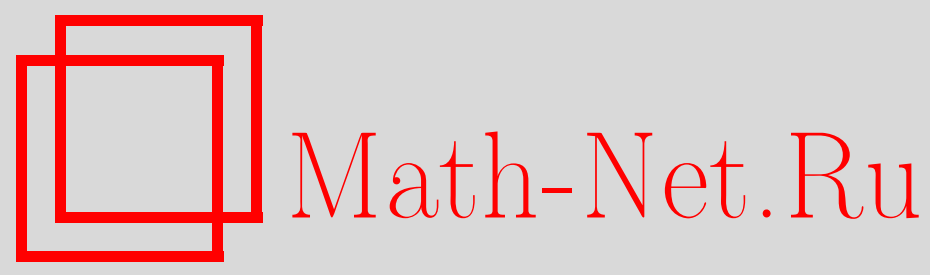

Ю. Ю. Кочетков, Соотношения и деформации нечетных гамильтоновых супералгебр, Матем. заметки, 1998, том 63, выпуск 3, 391-401

DOI: https://doi.org/10.4213/mzm1294

Использование Общероссийского математического портала Math-Net.Ru подразумевает, что вы прочитали и согласны с пользовательским соглашением http://www.mathnet.ru/rus/agreement

Параметры загрузки:

IP: 54.210 .77 .194

26 апреля 2023 г., 13:04:15

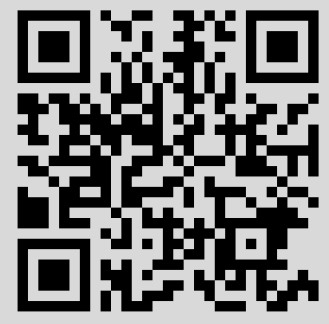




\title{
СООТНОШЕНИЯ И ДЕФОРМАЦИИ НЕЧЕТНЫХ ГАМИЛЬТОНОВЫХ СУПЕРАЛГЕБР
}

\author{
Ю. Ю. Кочетков
}

\begin{abstract}
Вычис ляются следующие (ко)гомологии супералгебры Ли $H(0, n): H^{2}(H, H)$-когомологии с коэффициентами в присоединенном модуле и $H_{2}(L H)$ - гомологии нильпотентной подалгебры. Показано, что $\operatorname{dim} H^{2}(H, H)=1$.

Библиография: 6 названий.
\end{abstract}

1. Введение. Деформации и соотношения алгебр и супералгебр Ли изучались в ряде недавних работ. Естественное предположение, что простые алгебры - жесткие, и что в нильпотентных подалгебрах классических алгебр векторных полей нет непростых соотношений (см. ниже) при достаточно большом количестве переменных, было опровергнуто в работах [1], [2]. В обеих упомянутых работах изучается гамильтонова алгебpa. В данной работе, как и в [3], подробно рассмотрен случай нечетной гамильтоновой супералгебры Ли. Результаты вычислений, представленные в [3], здесь уточняются, обобщаются и доказьваются. Отметим, что наши методы отличаются от методов, использованных в [4]-[6], но сходны с методами работы [2].

Основное поле - $\mathbb{Q}$. Пусть $\xi_{1}, \ldots, \xi_{n}$ - нечетные переменные. Мономы $\xi_{i_{1}} \cdots \xi_{i_{k}}$ мы будем обозначать заглавными $E$ с индексами. Если $E=\xi_{i_{1}} \cdots \xi_{i_{k}}$, то через $|E|$ обозначается длина $E$, т.е. $|E|=k$. С мономами мы будем обрашаться как с подмножествами множества $\left\{\xi_{1}, \ldots, \xi_{n}\right\}$, т.е. использовать операции " $\cup, \cap, \backslash$ " и обозначения " $\in, \subset$ " в очевидном смысле (если $E_{1} \cap E_{2} \neq \varnothing$, то $E_{1} E_{2}=0$, а операция $E_{1} \cup E_{2}$ не определена). Определим также операцию суммы двух мономов по модулю 2 как их симметрическую разность: $E_{1}+E_{2}=\left(E_{1} \backslash E_{2}\right) \cup\left(E_{2} \backslash E_{1}\right)$. Нечетной гамильтоновой супералгеброй $H(0, n)=H$ называется линейная оболочка мономов $E, 0<|E|<n$. Скобку мы определим на мономах и продолжим на всю алгебру билинейно: $\left[E_{1}, E_{2}\right] \neq 0$ только в случае $\left|E_{1} \cap E_{2}\right|=1$, при этом

$$
\left[E_{1}, E_{2}\right]=(-1)^{\left|E_{1}\right|} \frac{\partial E_{1}}{\partial \xi_{i}} \frac{\partial E_{2}}{\partial \xi_{i}}, \quad \text { где } \xi_{i}=E_{1} \cap E_{2} .
$$

Эта операция задает на $H$ структуру супералгебры Ли. Алгебра $H(0, n)$ простая и является удобной моделью при изучении гомологических свойств "настоящих" гамильтоновых алгебр $H(2 n)$.

Работа выполнена при поддержке Российского фонда фундаментальных исследований, грант № 95-01-01263, и Международного научного фонда, грант RO 4300. 
Нечетная гамильтонова супералгебра изоморфна супералгебре Ли гамильтоновых векторных полей на нечетном $n$-мерном суперпространстве. Точнее, пусть $\delta_{i}=\partial / \partial \xi_{i}$. Тогда моному $E$ мы сопоставляем векторноеполе $\delta_{i}\left(\partial E / \partial \xi_{i}\right)$. Это поле аннулирует дифференциальную форму $\sum_{i=1}^{n}\left(d \xi_{i}\right)^{2}$, т.е. является нечетньп гамильтоновьм полем, поскольку дифференциал нечетной переменной четен. Это соответствие является супераналогом канонического изоморфизма между алгеброй Ли гамильтоновых векторных полей и алгеброй Ли функций Гамильтона.

Линейная оболочка мономов $E$ таких, что $|E|>2$, является нильпотентной подсупералгеброй в $H$ и обозначается через $L H$. В этой работе будут описаны соотношения в $L H$ и деформации в $H$, т.е. будут вычислены вторая группа гомологий $H_{2}(L H)$ алгебры $L H$ и вторая группа когомологий $H^{2}(H, H)$ алгебры $H$ с коэффициентами в присоединенном модуле. Эти две задачи тесно связаны. Сначала введем необходимые определения.

Алгебра $H$ градуирована: градуировка вводится формулой $\operatorname{deg}(E)=|E|-2$, тогда $\operatorname{deg}\left(\left[E_{1}, E_{2}\right]\right)=\operatorname{deg}\left(E_{1}\right)+\operatorname{deg}\left(E_{2}\right)$. Кроме того, в $H$ имеется $n$ "нечетных" градуировок $w_{i}, i=1, \ldots, n$ :

$$
w_{i}(E)=\left\{\begin{array}{l}
1, \text { если } \xi_{i} \in E, \\
0 \text { в противном случае, }
\end{array} \quad w_{i}\left(\left[E_{1}, E_{2}\right]\right) \equiv w_{i}\left(E_{1}\right)+w_{i}\left(E_{2}\right)(\bmod 2) .\right.
$$

Все элементы, циклы, цепи, коциклы, гомологии и когомологии будут предполагаться однородньми относительно градуировок $\operatorname{deg}$ и $w_{i}, i=1, \ldots, n$. Набор значений $\left\{w_{i}(g)\right\}$ для однородного элемента (цепи, цикла и пр.) будет рассматриваться как моном и обозначаться $W$, т.е. $\xi_{i} \in W$, если $w_{i}(g)=1$. Все теоретико-множественные операции применимы к $W$.

Прежде чем формулировать основные результаты работы, сделаем замечание. Если цикл $c \in C_{2}(L H)$ имеет степень 2 , то он нетривиален. Такие циклы мы будем называть простыми. Непростые ииклы-этоциклы степени 3 и выше. Вторая группа непростых гомологий $L H$ будет обозначаться $\tilde{H}_{2}(L H)$.

ТЕОрема 1. Выполнено $\operatorname{dim}\left(\tilde{H}_{2}(L H)\right)=n$. Однородный нетривиальный иикл имеет степень 3, вес $(0, \ldots, 0,1,0, \ldots, 0)$ (единица на $i$-м месте, $i=1, \ldots, n) u$ имеет представителя $c=E \wedge \xi_{i} E$, где $|E|=3$.

Теорема 2. Выполнено $\operatorname{dim}\left(H^{2}(H, H)\right)=1$. Нетривиальный коцикл описывается следующей формулой: $c\left(E E_{1}, E E_{2}\right)=(-1)^{\left|E E_{1}\right|} E_{1} E_{2}$, где $|E|=3$.

ЗАмЕчАниЕ. Это утверждение сходно с результатом работы [4]. Формула из формулировки теоремы 2 аналогична формуле, определяющей скобку

$$
\left[\xi E_{1}, \xi E_{2}\right]=(-1)^{\left|\xi E_{1}\right|} E_{1} E_{2}
$$

ТЕорема 3. Определенный в теореме 2 коиикл с задает глобальную деформаuию $h$ :

$$
h\left(E_{1}, E_{2}\right)=c_{0}\left(E_{1}, E_{2}\right)+t c_{1}\left(E_{1}, E_{2}\right)+t^{2} c_{2}\left(E_{1}, E_{2}\right)+t^{3} c_{3}\left(E_{1}, E_{2}\right)+\cdots,
$$

әде $c_{0}\left(E_{1}, E_{2}\right)=\left[E_{1}, E_{2}\right], c_{1}-$ это кочикл $c$, а 2-коиепь $c_{i}$ определяется следующим образом:

$$
c_{i}\left(E E^{\prime}, E E^{\prime \prime}\right)=(-1)^{\left|E E^{\prime}\right|} E^{\prime} E^{\prime \prime}, \quad|E|=2 i+1
$$




\section{2. Соотношения.}

ДоКАЗАТЕЛЬСТво тЕоРемЫ 1 . Пусть $E_{1} \wedge E_{2}$-произвольная 2 -цеп, $3<\left|E_{1}\right| \leqslant\left|E_{2}\right|$, и пусть $\xi \notin E_{1}$. Вычисляя дифференциал $d\left(\xi E_{11} \wedge \xi E_{12} \wedge E_{2}\right)$, где $E_{11} \cup E_{12}=E_{1}$, получаем

$$
E_{1} \wedge E_{2} \sim \pm \xi E_{11} \wedge\left[\xi E_{12}, E_{2}\right] \pm \xi E_{12} \wedge\left[\xi E_{11}, E_{2}\right] .
$$

Поэтому любая 2-цепь может быть представлена как линейная комбинация 2-цепей $E_{1} \wedge E_{2}$ таких, что $|E|=3$. Из условий однородности следует, что надо рассмотреть четыре случая:

1) $\left|E_{1} \cap E_{2}\right|=0$

2) $\left|E_{1} \cap E_{2}\right|=1$

3) $\left|E_{1} \cap E_{2}\right|=2$;

4) $\left|E_{1} \cap E_{2}\right|=3$.

1) (Самьй трудный случай). Пусть $\left|E_{1}\right|+\left|E_{2}\right|<n$ и $\xi \notin E_{1} \cup E_{2}$. Тогда $\pm E_{1} \wedge E_{2}=$ $d\left(E_{1} \wedge \xi E^{\prime} \wedge \xi E^{\prime \prime}\right)$, где $E^{\prime} \cap E^{\prime \prime}=\varnothing$ и $E^{\prime} \cup E^{\prime \prime}=E_{2}$. Пусть теперь $\left|E_{1}\right|+\left|E_{2}\right|=n$. Рассмотрим следствия из соотношений вида $d\left(E_{1} \wedge E_{2} \wedge E_{3}\right)$, где $\left|E_{1}\right|=\left|E_{2}\right|=3$, $\left|E_{1} \cap E_{2}\right|=\left|E_{1} \cap E_{3}\right|=\left|E_{2} \cap E_{3}\right|=\left|E_{1} \cap E_{2} \cap E_{3}\right|=1$, и если $\xi_{i} \in E_{1}+E_{2}$, то $i \leqslant 6$. Имеем $\xi_{i} \xi_{j} \xi_{k} \wedge E^{\prime} \sim(-1)^{i+j+k} \xi_{1} \xi_{2} \xi_{3} \wedge E$ и $\xi_{i} \xi_{j} \xi_{k} \xi_{l} \wedge E^{\prime \prime} \sim(-1)^{i+j+k+l} 2 \cdot \xi_{1} \xi_{2} \xi_{3} \wedge E$. Соотношения вида

$$
\begin{aligned}
d\left(\xi_{1} \xi_{2} \xi_{7}\right. & \wedge \xi_{1} \xi_{3} \xi_{4} \wedge \xi_{1} \xi_{5} \xi_{6} \cdots-\xi_{2} \xi_{4} \xi_{5} \wedge \xi_{3} \xi_{5} \xi_{7} \wedge \xi_{1} \xi_{5} \xi_{6} \cdots \\
+\xi_{1} \xi_{2} \xi_{7} & \left.\wedge \xi_{3} \xi_{5} \xi_{7} \wedge \xi_{4} \xi_{6} \xi_{7} \cdots\right) \\
\quad=2 \cdot \xi_{3} \xi_{5} \xi_{7} & \wedge E_{1}+\xi_{1} \xi_{3} \xi_{4} \wedge E_{2}+\xi_{2} \xi_{4} \xi_{5} \wedge E_{3}-\xi_{1} \xi_{2} \xi_{3} \xi_{5} \wedge E_{4}
\end{aligned}
$$

позволяют распространить эти правила на все цепи вида $E_{1} \wedge E_{2}$, где $\left|E_{1}\right|=3$ или 4. Далее получаем, что $E_{1} \wedge E_{2} \sim \pm k \cdot \xi_{1} \xi_{2} \xi_{3} \wedge E$, где $k=\left|E_{1}\right|-2$. Но $E_{1} \wedge E_{2}= \pm E_{2} \wedge E_{1} \sim$ $l \cdot \xi_{1} \xi_{2} \xi_{3} \wedge E$, где $l=\left|E_{2}\right|-2$. Поэтому $\xi_{1} \xi_{2} \xi_{3} \wedge E \sim 0$.

2) Для доказательства теоремы в этом случае, достаточно доказать эквивалентность с точностью до множителя двух любых цепей $E_{1} \wedge E_{2} \sim E_{3} \wedge E_{4}$ одинаковой степени и веса таких, что $\left|E_{1}\right|=\left|E_{2}\right|=3$ и $\left|E_{1} \cap E_{2}\right|=\left|E_{3} \cap E_{4}\right|=1$. Пусть $\xi_{i} \in E_{1} \cap E_{2}$. Тогда из соотношений вида

$$
d\left(\xi_{i} \xi_{j} \xi_{k} \wedge \xi_{i} \xi_{j} \xi_{l} \wedge \xi_{j} \cdots\right)= \pm \xi_{i} \xi_{j} \xi_{k} \wedge \xi_{i} \cdots \pm \xi_{i} \xi_{j} \xi_{l} \wedge \xi_{i} \cdots
$$

следует, что $\xi_{i} \xi_{j} \xi_{k} \wedge \xi_{i} E^{\prime} \sim \pm \xi_{i} \xi_{l} \xi_{m} \wedge \xi_{i} E^{\prime \prime}$. Рассмотрение соотношений

$$
d\left(\xi_{i} \xi_{j} \xi_{k} \wedge \xi_{p} \xi_{q} \xi_{r} \wedge \xi_{i} \xi_{p} \cdots\right)= \pm \xi_{i} \xi_{j} \xi_{k} \wedge \xi_{i} \cdots \pm \xi_{p} \xi_{q} \xi_{r} \wedge \xi_{p} \cdots
$$

завершает доказательство теоремы 1 в этом случае.

3) Этот случай практически очевиден, так как

$$
\xi_{i} \xi_{j} \xi_{k} \wedge \xi_{i} \xi_{j} E= \pm \frac{1}{2} d\left(\xi_{i} \xi_{j} \xi_{k} \wedge \xi_{i} \xi_{j} \xi_{k} \wedge \xi_{k} E\right) .
$$

4) Рассмотрим цепь $c=\xi_{i} \xi_{j} \xi_{k} \wedge \xi_{i} \xi_{j} \xi_{k} E$. Если $|E|>1$, то $c= \pm d\left(\xi_{i} \xi_{j} \xi_{k} \wedge \xi \xi_{i} \xi_{j} \xi_{k} \wedge \xi E\right)$ $\sim 0$, где $\xi \notin E \cup \xi_{i} \xi_{j} \xi_{k}$. Если $|E|=1$, то, как легко убедиться, $E_{1} \wedge E_{1} \xi_{i}-$ нетривиальньй цикл и $E_{1} \wedge E_{1} \xi_{i} \sim E_{2} \wedge E_{2} \xi_{i}$ для любых мономов $E_{1}, E_{2},\left|E_{1}\right|=\left|E_{2}\right|=3$.

Доказательство теоремы 1 закончено. 


\section{3. Деформации.}

ДОКАЗАТЕЛЬСТво тЕОРЕМЫ 2. Доказательство представляет собой анализ большого числа отдельных случаев, некоторые из которых требуют тщательного рассмотрения. Для того чтобы упростить изложение, мы введем соглашения об обозначениях.

Соглашения. Напомним, что $W$ - это вес исследуемого коцикла. Если $|W|=k$, то мы будем считать, что $W=\xi_{1} \ldots \xi_{k}$. Моном $E=\xi_{i_{1}} \ldots \xi_{i_{k}}$ будет обозначаться $\left(i_{1}, \ldots, i_{k}\right)$. Через $c$ будет обозначаться исследуемый коцикл, через $c_{1}-1$-коцепь. Степень $\operatorname{deg}(c)$ однородного коцикла $c$ определяется как $\operatorname{deg}(c)=\operatorname{deg}\left(E_{3}\right)-\operatorname{deg}\left(E_{1}\right)-\operatorname{deg}\left(E_{2}\right)$, если $c\left(E_{1}, E_{2}\right)=\alpha E_{3}, \alpha \neq 0$. Когда мы задаем значения коцепи $c_{1}$ на некоторых мономах, то всегда предполагаем, что значения $c_{1}$ на остальных мономах равны 0 . Так как значение $c$ на паре мономов $E_{1}, E_{2}$ есть моном с некоторым коэффициентом, мы будем писать $c\left(E_{1}, E_{2}\right)$ вместо коэффициента там, где это не приводит к непониманию.

Нашей целью будет построение коцикла $\tilde{c} \sim c$ такого, что $\tilde{c}\left(E_{1}, E_{2}\right)=0$ при $\left|E_{1}\right|=1$, если $\operatorname{deg}(c) \geqslant 0$, и при $\left|E_{1}\right| \leqslant 2$, если $\operatorname{deg}(c)<0$. Для этого нам понадобится процедуpa A.

ПроцеДУРА А. Пусть $\operatorname{deg}(c)=l>-(k-2) u c(i, E)=0$ для всех $i$ u всех мономов $E,|E|<k$. Тогда существует әквивалентный коиикл $\tilde{c} \sim c$ такой, что $\tilde{c}(i, E)=0,1 \leqslant i \leqslant n,|E|=k$.

ДокАЗАТЕЛЬСтво. Пусть $|E|=k$ и $c(i, E)=\alpha E_{1}, \alpha \neq 0$. Тогда $\left|E_{1}\right|>1$. Если $i \in E_{1}$, то из $d c(i, i, E)= \pm 2\left[i, E_{1}\right]$ следует, что $\alpha=0$. Поэтому $i \notin E_{1}$. Пусть $E_{2}=i \cup E_{1}$. Тогда $c(j, E)=0$, если $j \notin E_{2}$. В самом деле, пусть $c(j, E)=\beta E_{3}, \beta \neq 0$. Имеем $i+E+E_{1}=W, j+E+E_{3}=W$; значит, $i+j=E_{1}+E_{3}$. Но $i \notin E_{1}, j \notin E_{1}$. Следовательно, $E_{3}=E_{1} \cup i \cup j$, что противоречит однородности $c$ по степени. Пусть $j \in E_{2}$ и $c(j, E)=\beta\left(E_{2} \backslash j\right)$, тогда

$$
d c(i, j, E)= \pm[i, c(j, E)] \pm[j, c(i, E)]= \pm \beta E_{1} \pm \alpha E_{1}=0 .
$$

Эти равенства дают возможность определить значение 1-коцепи $c_{1}(E)=\gamma E_{2}$ так, чтобы $d c_{1}(i, E)=c(i, E)$ для всех $i$. Теперь заменим $c$ на эквивалентный коцикл $\tilde{c}=c-d c_{1}$. Тогда $\tilde{c}(i, E)=0$. Отметим, что $\tilde{c}\left(i, E_{1}\right)=0$, если $\left|E_{1}\right| \leqslant k$ и $c\left(i, E_{1}\right)=0$. Продолжаем построение.

Если $\operatorname{deg}(c) \geqslant 0$, то также будет использоваться процедура Б.

ПроцеДУРА Б. Пусть $\operatorname{deg}(c) \geqslant 0 u c(i, E)=0$ для всех $i$ u всех E. Тогда $c=0$.

ДокаЗАтЕЛЬСтво. Пусть $c\left(E_{1}, E_{2}\right)=\alpha E_{3}, \alpha \neq 0$, где $2 \leqslant\left|E_{1}\right| \leqslant\left|E_{2}\right|$. Тогда $\left|E_{3}\right| \geqslant 2$. Если $i \in E_{3}$, то

$$
d c\left(i, E_{1}, E_{2}\right)= \pm c\left(\left[i, E_{1}\right], E_{2}\right) \pm c\left(E_{1},\left[i, E_{2}\right]\right) \pm \alpha\left[i, E_{3}\right]=0 .
$$

Так как $\left|\left[i, E_{1}\right]\right|+\left|E_{2}\right|=\left|E_{1}\right|+\left|\left[i, E_{2}\right]\right|<\left|E_{1}\right|+\left|E_{2}\right|$, мы можем проводить индукцию по $\left|E_{1}\right|+\left|E_{2}\right|$, начиная с $\left|E_{1}\right|+\left|E_{2}\right|=4$.

СлЕДСтвиЕ. Пусть $\operatorname{deg}(c)=k<0$ u $c(i, E)=0$ для всех $i$ u $E$, u $c((i, j), E)=0$ для всех $i, j$ и всех $E,|E|=-k+1$. Тогда $c((i, j), E)=0$ для всех $i, j$ и всех $E$. 
ДоКАЗАТЕЛЬСТВо ТЕОРЕМЫ 2. Мы последовательно рассмотрим четыре случая:

1) $\operatorname{deg}(c)>0$;

2) $\operatorname{deg}(c)=0$

3) $\operatorname{deg}(c)=-1$;

4) $\operatorname{deg}(c)<-1$.

1) Здесь нам достаточно доказать существование эквивалентного коцикла $\tilde{c}$ такого, что $\tilde{c}(i, j)=0$, а затем использовать процедуры А и Б. $Е с л и \operatorname{deg}(c)=1$, то $|W|=1$ или 3.

a) Случай $|W|=3$ возможен только если $c(1,2)=\alpha \cdot 3 ; c(1,3)=\beta \cdot 2$ и $c(2,3)=\gamma \cdot 1$ могут быть отличны от 0 . Из $d c((1,2), 1,3)=d c((1,2), 1,1)=0$ следует, что $\alpha+\beta+\gamma=0$. Определим 1-коцепь $c_{1}: c_{1}(1)=\lambda \cdot(2,3), c_{1}(2)=\mu \cdot(1,3), c_{1}(3)=\nu \cdot(1,2)$. Тогда $d c_{1}(1,2)=-(\lambda+\mu) \cdot 3, d c_{1}(1,3)=(\lambda-\nu) \cdot 2, d c_{1}(2,3)=(\mu+\nu) \cdot 1$, т.е. мы можем подобрать коэффициенты $\lambda, \mu, \nu$ так, чтобы $d c_{1}(i, j)=c(i, j)$ для всех $i, j$. Теперь заменим $c$ на эквивалентный коцикл $c-d c_{1}$.

б) Если $|W|=1$, то $c(1, i)=\alpha_{i} \cdot i, i>1$, и $c(i, i)=\beta_{i} \cdot 1,1 \leqslant i \leqslant n$, могут быть отличны от 0. Определим 1-коцепь $c_{1}(i)=-\alpha_{i} \cdot(1, i), i>1$. При этом $d c_{1}(1, i)=c(1, i)$. Пусть $\tilde{c}=c-d c_{1}$. Тогда $d \tilde{c}((1, i), i, i)=-2 \tilde{c}(1, i)-\tilde{c}(i, i)=0$. Так как $\tilde{c}(1, i)=0$, имеем $\tilde{c}(i, i)=0$.

Если $\operatorname{deg}(c)>1$ и $c\left(i_{0}, j_{0}\right)=\alpha \cdot E \neq 0$, то $|W|=|E|+2,|E|$ или $|E|-2$.

a) Если $|W|=|E|+2$, то $c(i, j) \neq 0$ только в случае $i \in W, j \in W, i \neq j$. При этом $c(i, j)=\alpha_{i j}(W \backslash(i, j))$. Равенства $d c(i, j, k)=0, i<j<k$, дают соотношения

$$
(-1)^{i-1} \alpha_{j k}+(-1)^{j-2} \alpha_{i k}+(-1)^{k-3} \alpha_{i j}=0,
$$

среди которых есть $(|W|-1)(|W|-2) / 2$ независимых:

$$
\alpha_{i j}+(-1)^{i-2} \alpha_{1 i}+(-1)^{j-3} \alpha_{1 j}=0, \quad 1<i<j .
$$

Таким образом, среди $\alpha_{i j}$ есть $|W|-1$ свободных. Зададим 1 -коцепь $c_{1}(i)=\beta_{i}(W \backslash i)$, $i \in W$. Тогда

$$
d c_{1}(i, j)= \pm\left((-1)^{i-1} \beta_{j}+(-1)^{j-2} \beta_{i}\right)(W \backslash(i, j)), \quad i<j .
$$

Размерность пространства этих соотношений равна $|W|-1$. Базис образуют соотношения $\beta_{j}+(-1)^{j-2} \beta_{1}$. Следовательно, можно подобрать $\beta_{i}$ так, чтобы выполнялось равенство $d c_{1}(i, j)=c(i, j)$ для всех $i, j$. Теперь, как и вьше, положим $\tilde{c}=c-d c_{1}$.

б) Если $|W|=|E|$, то $c(i, j)$ может быть отлично от 0 только в случае $i \in W, j \notin W$, или $i=j$. При этом $c(i, j)=\alpha_{i j}((W \cup j) \backslash i)$ в первом случае и $c(j, j)=\alpha_{j} \cdot W-$ во втором. Из $d c(i, i, i)=0$ следует, что $c(i, i)=0$, если $i \in W$. Тогда соотношения $d c(i, j, j)=\left( \pm \alpha_{j} \pm 2 \alpha_{i j}\right) W=0, i \in W, j \notin W$, позволяют определить 1-коцепь $c_{1}(j)=\beta_{j}(W \cup j)$ так, чтобы $d c_{1}(i, j)=c(i, j)$.

в) Если $|W|=|E|-2$, то $c(i, j)$ может быть отличным от 0 только в случае $i \notin W$, $i \neq j$. При этом $c(i, j)=\alpha_{i j}(W \cup i \cup j)$. Но тогда $d c(i, j, j)= \pm 2 \alpha_{i j}(W \cup i)=0$.

2) Здесь нам достаточно построить эквивалентный коцикл $\tilde{c}$ такой, что $\tilde{c}(i,(j, k))=0$ для всех $i, j, k$, и воспользоваться процедурой А. Пусть $c\left(i_{0},\left(j_{0}, k_{0}\right)\right) \neq 0$. Тогда $|W|=0$, 2 или 4.

a) Если $|W|=4$, то $c(i,(j, k))$ может быть отлично от 0 только в случае, когда $i, j, k$ различны и $1 \leqslant i, j, k \leqslant 4$. Тогда $c(i,(j, k))=\alpha(W \backslash(i, j, k))$. Имеем $d c(3,(3,4),(1,2))=$ $-c(4,(1,2))-c(3,(1,2))=0$. Теперь мы можем определить 1 -коцепь $c_{1}((1,2))=\beta(3,4)$ 
так, чтобы $d c_{1}(3,(1,2))=c(3,(1,2)), d c_{1}(4,(1,2))=c(4,(1,2))$. Аналогично определяем значение $c_{1}$ на других мономах $(i, j), 1 \leqslant i, j \leqslant 4$.

б) В случае $|W|=2$ могут быть отличны от 0 только следуюшие значения $c: c(i,(1,2))$, $c(i,(1, i)), c(i,(2, i)), c(2,(1, i)), c(1,(2, i))$. Отметим, что из $d c(i, i,(1,2, i))=0$ следует, что $c(i,(1,2))=0$ при $i>2$. Мы будем определять значения 1 -коцепи $c_{1}(1)=\gamma_{1} \cdot 2$, $c_{1}(2)=\gamma_{2} \cdot 1, c_{1}((1, i))=\alpha_{i}(2, i)$ и $c_{1}((2, i))=\beta_{i}(1, i), i>2$, так, чтобы система

$$
\begin{aligned}
d c_{1}(i,(1, i)) & =\left(\gamma_{1}-\alpha_{i}\right)(2)=c(i,(1, i)), \quad i>2 \\
d c_{1}(i,(2, i)) & =\left(\gamma_{2}-\beta_{i}\right)(1)=c(i,(2, i)), \quad i>2 \\
d c_{1}(1,(1,2)) & =\left(-\gamma_{2}-\gamma_{1}\right)(2)=c(1,(1,2)) \\
d c_{1}(2,(1,2)) & =\left(\gamma_{1}+\gamma_{2}\right)(1)=c(2,(1,2))
\end{aligned}
$$

имела решения в переменных $\alpha, \beta$ и $\gamma$. Ранг левой части системы равен $2 n-3$, но и ранг правой части такой же, так как существует нетривиальное соотношение

$d c(3,(1,3),(1,2))+d c(3,(2,3),(1,2))-d c((1,2),(1,3),(2,3))=-c(2,(1,2))-c(1,(1,2))$.

Таким образом, мы можем определить эквивалентньй коцикл $c-d c_{1}$, которьй мы также будем обозначать $c$, такой, что только $c(1,(2, i))$ и $c(2,(1, i)), i>2$, могут быть отличны от 0 . Но из равенств

$$
\begin{aligned}
& d c(2,(1,2),(2, i))=c(1,(2, i))+c(2,(1, i))+c((1,2),(2, i))=0 \\
& d c(i,(1,2),(2, i))=-c((1,2),(2, i))=0 \\
& d c(i,(1, i),(2, i))=c(1,(2, i))-c(2,(1, i))=0
\end{aligned}
$$

следует, что $c(i,(j, k))=0$ для всех $i, j, k$.

в) В случае $|W|=0$ только $c(i,(i, j))=\alpha \cdot j$ может быть отлично от 0. Мы будем последовательно строить эквивалентный коцикл, обладающий нужными свойствами. Прямое вычисление показывает, что существует коцикл $\tilde{c}$ такой, что $\tilde{c}(i,(i, j))=0$ при $1 \leqslant i, j \leqslant 3$. Пусть уже построен коцикл $\tilde{c}$, для которого $\tilde{c}(i,(i, j))=0$ при $1 \leqslant i, j<k$. Докажем существование 1 -коцепи $c_{1}$ такой, что $d c_{1}(i,(i, k))=\tilde{c}(i,(i, k))$, $i<k, d c_{1}(k,(i, k))=\tilde{c}(k,(i, k)), i<k$, и $d c_{1}(i,(i, j))=0,1 \leqslant i, j<k$. Пусть $c_{1}((i, k))=\alpha_{1}(i, k), c_{1}(k)=\alpha \cdot k$. Тогда

$$
d c_{1}(i,(i, k))=\left(-\alpha+\alpha_{i}\right) \cdot k, \quad d c_{1}(k,(i, k))=\left(-\alpha-\alpha_{i}\right) \cdot i .
$$

Так как

$d(\tilde{c}((1, i),(1, k), k)+\tilde{c}((1, i),(1, k), i)=-\tilde{c}(i,(i, k)+\tilde{c}(1,(1, k))+\tilde{c}(k,(1, k))-\tilde{c}(k,(i, k))=0$, размерность пространства $\{\tilde{c}(i,(i, k)), \tilde{c}(k,(i, k))\}_{i<k}$ равна $k$. Таким образом, система

$$
-\alpha+\alpha_{i}=\gamma_{i}, \quad-\alpha-\alpha_{i}=\delta_{i},
$$

где $\tilde{c}(i,(i, k))=\gamma_{i} \cdot k, \tilde{c}(k,(i, k))=\delta_{i} \cdot i$, разрешима. Переходя к коциклу $\tilde{c}-d c_{1}$, продолжаем индуктивньй процесс.

3) Здесь наша задача состоит в том, чтобы построить эквивалентный коцикл $\tilde{c}$ такой, что $\tilde{c}(i, E)=0,|E|=3, \tilde{c}\left(E_{1}, E_{2}\right)=0,\left|E_{1}\right|=\left|E_{2}\right|=2$. После чего можно использовать процедуры А и Б. Пусть $c(i, E) \neq 0$ для некоторого $i$ и некоторого монома $E,|E|=3$, или $c\left(E_{1}, E_{2}\right) \neq 0$ для некоторых мономов $E_{1}, E_{2},\left|E_{1}\right|=\left|E_{2}\right|=2$. Тогда $|W|=1,3$ или 5. 
a) Если $|W|=5$, то $c(i, E)=\alpha \cdot j, E \cup i \cup j=W$. Имеем $d c(E, i,(i, j))=c(j, E)+$ $c(i, E)=0$. Значит, можно определить $c_{1}(E)=\beta(i, j)$ так, чтобы $d c_{1}(i, E)=c(i, E)$, $d c_{1}(j, E)=c(j, E)$. Перейдем к эквивалентному коциклу $\tilde{c}=c-d c_{1}$. Пусть $\left|E_{1}\right|=$ $\left|E_{2}\right|=2$ и $E_{1} \cup E_{2} \cup i=W$. Тогда $d \tilde{c}\left(i,\left(i, E_{1}\right), E_{2}\right)=-\tilde{c}\left(E_{1}, E_{2}\right)=0$.

б) В случае $|W|=3$ имеем

$$
d c((1,2),(3, i),(i, j))=c((1,2),(3, j))-c((1,2),(3, i))=0,
$$

что дает нам возможность определить $c_{1}((1,2))=\alpha \cdot 3$ так, чтобы $d c_{1}((1,2),(3, i))=$ $c((1,2),(3, i))$ при $i>3$. Для $c((1,3),(2, i))$ и $c((2,3),(1, i))$ проводим аналогичные утверждения. Переходим к эквивалентному коциклу $c-d c_{1}$, который также будет обозначаться $c$. Имеем

$d c((1,2),(1,3),(1, i))=c((2,3),(1, i))+c((1,3),(2, i))-c((1,2),(3, i))-c((1,2),(1,3))=0$, т.е. $c((1,2),(1,3))=0$. Аналогично получаем, что

$$
c((1,2),(3, i))=c((1,3),(2, i))=c((2,3),(1, i))=0
$$

при всех $i$. Далее,

$$
d c((1, i),(2, i),(3, j))=c((1,2),(3, j))-c((1, i),(2, i))+c((1, i),(2, i))=0,
$$

т.е. $c\left(E_{1}, E_{2}\right)=0$ для всех $E_{1}, E_{2},\left|E_{1}\right|=\left|E_{2}\right|=2$.

Рассмотрим теперь $c(i, E),|E|=3$. Здесь отличными от 0 могут быть $c(i,(1,2,3))$; $c(i, E), i>3, i \in E,|E \cap(1,2,3)|=2 ; c(i, E), i \leqslant 3, i \notin E,|E \cap(1,2,3)|=2$. Из соотношений

$$
\begin{aligned}
& d c((1,2,4), 4,(3,5))=-c((1,2,4),(3,5))-c(4,(1,2,4))=0 \\
& d c((1,2,4), 5,(3,5))=c(3,(1,2,4))+c((1,2,4),(3,5))=0
\end{aligned}
$$

получаем, что $c(3,(1,2,4))+c(4,(1,2,4))=0$, т.е. мы можем определить значение 1 -коцепи $c_{1}((1,2,4))=\alpha(3,4)$ так, чтобы $d c_{1}(3,(1,2,4))=c(3,(1,2,4)), d c_{1}(4,(1,2,4))=$ $c(4,(1,2,4))$. Аналогично определяем $c_{1}(E),|E \cap(1,2,3)|=2$. Переходя к эквивалентному коциклу $\tilde{c}$, имеем $\tilde{c}(i, E)=0$, если $|E \cap(1,2,3)|=2$. Далее, $d \tilde{c}(i, i,(1,2,3, i))=$ $-2 \tilde{c}(i,(1,2,3))=0$, а из соотношений типа

$$
\begin{aligned}
& d \tilde{c}(4,(3,4),(1,2,3))=\tilde{c}(3,(1,2,3))+\tilde{c}((3,4),(1,2,3))=0 \\
& d \tilde{c}(3,(3,4),(1,2,3))=\tilde{c}(3,(1,2,3))-\tilde{c}((3,4),(1,2,3))=0
\end{aligned}
$$

следует, что $\tilde{c}(i, E)=0$ для всех $i$ и всех $E,|E|=3$.

в) В случае $|W|=1$ только $c(i,(1, i, j))=\alpha \cdot j$ и $c((1, i),(i, j))=\beta \cdot j$ могут быть отличны от 0. Имеем

$$
\begin{aligned}
& d c((1, i),(i, j),(j, k))=-c((1, j),(j, k))+c((1, i),(i, k))-c((1, i),(i, j))=0 \\
& d c((1, i),(i, k),(j, k))=-c((1, k),(j, k))-c((1, i),(i, j))+c((1, i),(i, k))=0
\end{aligned}
$$

откуда $c((1, j),(j, k))=c((1, k),(j, k))$. Далее,

$$
d c((1,2),(2, i),(i, j))=-c((1, i),(i, j))+c((1,2),(2, j))-c((1,2),(2, i))=0 .
$$


Пусть $c_{1}((1, i))=\beta_{i} \cdot i, i>1$. Тогда система $d c_{1}((1,2),(2, i))=c((1,2),(2, i))$, т.е. $-\beta_{i}+\beta_{2}=\alpha_{i}$, разрешима. Следовательно, переходя к эквивалентному коциклу $\tilde{c}=$ $c-d c_{1}$, имеем $\tilde{c}((1, i),(i, j))=0$. Пусть теперь $i<j$. Тогда

$$
d \tilde{c}(i,(1, i, j),(i, j))=\tilde{c}(j,(1, i, j))+\tilde{c}(i,(1, i, j))=0,
$$

т.е. мы можем определить $c_{1}((1, i, j))=\beta_{i j}(i, j)$ так, чтобы свойства $d c_{1}(i,(1, i, j))=$ $\tilde{c}(i,(1, i, j)), d c_{1}(j,(1, i, j))=\tilde{c}(j,(1, i, j))$ были вьполнены.

4) Наша задача - построить эквивалентньй цикл $\tilde{c}$ такой, что $\tilde{c}(i, E)=0,|E|=k$, и $\tilde{c}\left(E_{1}, E_{2}\right)=0,\left|E_{1}\right|=2,\left|E_{2}\right|=k-1$. Если $c(i, E) \neq 0,|E|=k$, или $c\left(E_{1}, E_{2}\right) \neq 0$, $\left|E_{1}\right|=2,\left|E_{2}\right|=k-1$, то $|W|=k+2, k, k-2$ или $k-4$.

a) Случай $|W|=k+2$ разбирается так же, как случай 3 a).

б) В случае $|W|=k$ пусть $c((i, j), E) \neq 0,|E|=k-1$. Тогда $|E \cap W|=k-1$ или $k-2$.

Если $|E \cap W|=k-2,(i, j)=W \backslash E$, то $c((i, j), E)=\alpha(E \backslash W)$. Пусть $l, m \in E \cap W$ и $B=E \backslash(l, m)$. Тогда

$$
\begin{aligned}
d c((i, j),(i, l),(i, m, B))= & c((j, l),(i, m, B))+c((i, l),(j, m, B))-c((i, j),(l, m, B))=0, \\
d c((i, j),(j, l),(j, m, B))= & -c((i, l),(j, m, B))-c((j, l),(i, m, B)) \\
& -c((i, j),(l, m, B))=0
\end{aligned}
$$

и $\alpha=0$.

Если $E \in W$ и $i=W \backslash E, j, l \notin W$, то

$$
d c((i, j),(j, l), E)=-c((i, l), E)+c((i, j), E)=0,
$$

т.е. мы можем определить $c_{1}(E)=\alpha \cdot i$ так, чтобы $d c_{1}((i, j), E)=c((i, j), E)$ для всех $j \notin W$, и т.д. Пусть теперь $j \in W, l \notin W$. Тогда

$$
\begin{aligned}
d c((i, j),(j, l), E)= & -c((i, l), E) \pm c((j, l),(E \backslash j) \cup i) \\
& \pm c((i, j),(E \backslash j) \cup l)+c((i, j), E)=0 .
\end{aligned}
$$

Так как первые три члена в правой части равенства равны 0 , то и $c((i, j), E)=0$.

Если $|E \cap W|=k-2, i \in W \backslash E, j \in E \backslash W$, то $c((i, j), E)=\alpha(W \backslash(E \cup i))$. Докажем, что $\alpha=0$. Проиллюстрируем логику рассуждений на примере $k=4, i=1, j=5$, $E=(2,3,5)$. Имеем, как и выше,

$$
\begin{aligned}
& d c((1,5),(2,3),(3,4,5))=c((1,5),(2,4,5))+c((1,5),(3,4,5))=0, \\
& d c((2,5),(1,3),(3,4,5))=c((2,5),(1,4,5))+c((2,5),(3,4,5))=0, \\
& d c((1,2),(3,5),(1,4,5))=c((3,5),(2,4,5))+c((3,5),(1,4,5))=0, \\
& d c((1,5),(2,5),(3,4,5))=c((1,5),(3,4,5))-c((2,5),(3,4,5))=0, \\
& d c((1,5),(3,5),(2,4,5))=c((1,5),(2,4,5))-c((3,5),(2,4,5))=0, \\
& d c((2,5),(3,5),(1,4,5))=c((2,5),(1,4,5))-c((3,5),(1,4,5))=0,
\end{aligned}
$$

откуда $c((i, j), E)=0$.

В случае $E \subset W,|W|=n$, приведенные выше рассуждения не проходят, и эквивалентньй коцикл $\tilde{c}$ с требуемыми свойствами построить не удается. Поэтому мы проведем прямое доказательство тривиальности $c$ в этом случае.

При $n=6$ это доказывается прямым вычислением. Пусть $n>6$. Тогда $c\left(E_{1}, E_{2}\right)$ равно линейной комбинации $c\left(E^{\prime}, E^{\prime \prime}\right),\left|E^{\prime}\right| \leqslant 3$. Рассмотрим множество $\left\{c\left(E_{1}, E_{2} E^{\prime}\right)\right\}$, где $E_{1}, E_{2} \subset\{1,2,3,4,5,6\}$, а $E^{\prime}=\{7, \ldots, n\}$. Используя тривиальность $c$ при $n=6$, 
можно перейти к эквивалентному коциклу г такому, что $\tilde{c}\left(E_{1}, E_{2}\right)=0$ при $E_{1} \subset\{1, \ldots, 6\}$, $\left|E_{1}\right|<4$. Рассмотрим $c\left(E^{\prime}, E^{\prime \prime}\right)$, где $\left|E^{\prime}\right| \leqslant 4,\left|E^{\prime} \cap\{7, \ldots, n\}\right|=1$. Пусть $E^{\prime}=(1,7)$, $E^{\prime \prime}=(1,2,3,4,5,6)$. Имеем

$$
\begin{aligned}
d c((1,7),(1,2,7),(3,4,5,6,7))= & c((1,2,7),(1,3,4,5,6))+c((1,7),(1,2,3,4,5,6)) \\
& +c((1,2,7),(3,4,5,6,7))=0 .
\end{aligned}
$$

Tak как

$$
d c((1,2,7),(1,2,3),(2,4,5,6))= \pm c((1,2,7),(1,3,4,5,6))=0,
$$

имеем $c((1,7),(1,2,3,4,5,6)=-c((1,2,7),(3,4,5,6,7))$. Далее,

$$
\begin{gathered}
d c(2,(1,2,7),(2,3,4,5,6,7))=c((1,7),(2,3,4,5,6,7))+c((1,2,7),(3,4,5,6,7)) \\
\pm c((1,2,7),(2,3,4,5,6,7))=0 .
\end{gathered}
$$

Из $d c(7,(1,2,7),(2,3,4,5,6,7))= \pm c((1,2,7),(2,3,4,5,6,7))=0$ следует, что $c((1,2,7)$, $(3,4,5,6,7))=-c((1,7),(2,3,4,5,6,7))$. Далее, соотношения вида

$$
d c((1,2),(1,7),(1,2,3,4,5,6))=0
$$

дают

$$
c((1,7),(1,2,3,4,5,6))=c((2,7),(1,2,3,4,5,6)) .
$$

Из этих равенств следует, что мы можем определить $c_{1}((1,2,3,4,5,6))=\alpha \cdot 7$ так, чтобы $d c_{1}\left(E_{1}, E_{2}\right)=c\left(E_{1}, E_{2}\right)$, где $\left|E_{1}\right|<4,7 \in E_{1}$ и $E_{1} \subset\{1, \ldots, 7\}$. Отметим, что если $E_{1} \subset\{1, \ldots, 6\}$, то $d c_{1}\left(E_{1}, E_{2}\right)=0$. Теперь мы можем переходить к следующему шагу.

Рассмотрим $c(i, E),|E|=k$. Если $c(i, E) \neq 0$, то либо $E=W$, либо $|E \cap W|=k-1$. Тогда либо $i=W \backslash E$, либо $i=E \backslash W$.

Рассмотрим случай $E=W, i \in W$. Пусть, например, $i=1$. Тогда

$$
d c(1,(1,2, k+1),(3, \ldots, k, k+1))= \pm c(1, E)=0 .
$$

Рассмотрим случай $|E \cap W|=k-1$. Пусть, например, $E=(2, \ldots, k, k+1)$. Тогда

$$
d c(1,(1, k+1), E)=-c(k+1, E)-c(1, E)=0
$$

что дает возможность определить $c_{1}(E)=\alpha(1, k+1)$.

В случае $E=W, i \notin W$, пусть $i=k+1$. Тогда

$$
d c(k+1,(1, k+1),(2, \ldots, k, k+1))= \pm c(k+1, E)=0 .
$$

в) В случае $|W|=k-2$ отличными от 0 могут быть только следующие значения коцикла: $c(i,(W \cup i \cup j)), i \notin W, j \notin W ; c((i, j),(W \cup i)), i \notin W ; c\left((i, j),\left(W^{\prime} \cup i \cup j\right)\right)$, $W^{\prime} \subset W,\left|W^{\prime}\right|=k-3, i \notin W, j \notin W ; c\left((i, m),\left(W^{\prime} \cup i \cup j\right)\right), m=W \backslash W^{\prime}$. Здесь рассуждения почти такие же, как в случае 3 в); дополнительно необходимо рассмотреть только $c\left((i, j),\left(W^{\prime} \cup i \cup j\right)\right)$. Имеем $(l \notin W)$ :

$$
d c\left((i, j),(m, l),\left(W^{\prime} \cup i \cup j\right)\right)=c\left((i, j),\left(W^{\prime} \cup i \cup j\right)\right)=0 .
$$

г) В случае $|W|=k-4$ имеем $c(i, E)=0$ при $|E|=k$, и надо рассмотреть только $c((i, j), E),|E|=k-1$. Здесь отличными от 0 могут быть только $c((i, j),(W \cup i \cup j \cup l))$, где $i, j, l \notin W$. Но тогда

$$
d c((i, j),(i, j, m),(W \cup l \cup m))= \pm c((i, j),(W \cup i \cup j \cup l))=0,
$$


если $|W|>0$, и

$$
d c((i, j),(i, j, l),(i, j, l))= \pm 2 c((i, j),(i, j, l))=0
$$

если $|W|=0$.

Таким образом, мы построили эквивалентньй коцикл, которьй мы также будем обозначать $c$, такой, что $c(i, E)=0,|E|=k$, и $c((i, j), E)=0,|E|=k-1$. Если $\operatorname{deg}(c)=-2$, то применение процедур А и Б показьвает, что $c$ тривиален. В случае $\operatorname{deg}(c)<-2$ поступаем следующим образом. Если $c\left(E_{1}, E_{2}\right)=0$ для всех пар $\left\{E_{1}, E_{2}\right\}$, $2<\left|E_{1}\right| \leqslant\left|E_{2}\right|,\left|E_{1}\right|+\left|E_{2}\right|=k+1$, то процедура Б дает $c=0$. Пусть $c\left(E_{1}, E_{2}\right)=\alpha \cdot i$, $\alpha \neq 0$. Зафиксируем $i$. Рассмотрим множество таких пар, что $E_{1}+E_{2} \equiv W \backslash i$. Определим на этом множестве 2-коцепь $\bar{c}$ с тривиальными коэффициентами $\bar{c}\left(E_{1}, E_{2}\right)=\alpha$, если $c\left(E_{1}, E_{2}\right)=\alpha \cdot i$. Тогда $\bar{c}-$ это ненулевой коцикл на $L H$. Из доказательства теоремы 1 следует, что существуют мономы $E_{1}, E_{2},\left|E_{1}\right|=3$, такие, что $\bar{c}\left(E_{1}, E_{2}\right) \neq 0$. Тогда возможны следующие случаи:

i) $i \notin E_{2},\left|E_{2}\right|<n-1$;

ii) $i \notin E_{2},\left|E_{2}\right|=n-1$;

iii) $i \in E_{2}, i \notin E_{1}$;

iv) $i \in E_{1}, i \in E_{2}$

(для краткости мы будем писать $i E$ вместо $i \cup E$ ).

i) Имеем $\left.d c\left(i, E_{1}, i E_{2}\right)\right)= \pm c\left(E_{1}, E_{2}\right)=0$.

ii) Здесь либо $i \in E_{1}$, либо $i \notin E_{1}$. Если $i \in E_{1}$, то $d c\left(E_{1}, E_{1}, E_{2}\right)= \pm 2 c\left(E_{1}, E_{2}\right)=0$. Если $i \notin E_{1}$, то $E_{1} \subset E_{2}$ и $d c\left(i, i E_{1}, E_{2}\right)=-c\left(E_{1}, E_{2}\right)=0$.

iii) Пусть существует $j, j \in E_{1}, j \notin E_{2}$. Тогда $E_{1}=j E^{\prime}, E_{2}=i E^{\prime \prime}$ и

$$
d c\left((i, j), i E^{\prime}, i E^{\prime \prime}\right)=c\left(E_{1}, E_{2}\right)+c\left(i E^{\prime}, j E^{\prime \prime}\right)+c\left(i E^{\prime}, i E^{\prime \prime}\right)=0 .
$$

Так как второе и третье слагаемые в правой части равны 0 в силу i) и іi), выполнено $c\left(E_{1}, E_{2}\right)=0$. Пусть теперь $E_{1} \subset E_{2}$ и $\left|E_{2}\right|>4$. Тогда существует $j, j \in E_{2}, j \notin E_{1}$ и $d c\left((i, j), E_{1}, E_{2}\right)=c\left(E_{1}, E_{2}\right)=0$. Если $\left|E_{2}\right|=4$, то мы получаем нетривиальный коцикл.

iv) Пусть существует $j, j \notin E_{1}, j \notin E_{2}$, и $E_{1}=i E^{\prime}, E_{2}=i E^{\prime \prime}$. Тогда

$$
d c\left((i, j), i E^{\prime}, i E^{\prime \prime}\right)=c\left(j E^{\prime}, i E^{\prime \prime}\right)+c\left(i E^{\prime}, j E^{\prime \prime}\right)-c\left(i E^{\prime}, i E^{\prime \prime}\right)=0 .
$$

Так как первые два слагаемых в правой части равны 0 в силу i), имеем $c\left(E_{1}, E_{2}\right)=0$. Если такого $j$ нет, но $\left|E_{1} \cap E_{2}\right|>1$, то $d c\left(E_{1}, E_{1}, E_{2}\right)= \pm 2 c\left(E_{1}, E_{2}\right)=0$. Случай $\left|E_{1} \cap E_{2}\right|=i$ рассмотрен вьше.

Мы доказали, что только коцикл степени -4 и веса 0 может быть нетривиальным. Прямая проверка показьвает, что условие $c(E, E i)=-i$ порождает нетривиальньй коцикл $c\left(E E_{1}, E E_{2}\right)=(-1)^{\left|E E_{1}\right|} E_{1} E_{2}$. Доказательство теоремы 2 закончено.

ЗАмЕчАнИЕ. Если основньм полем является $\mathbb{C}$, то можно ввести новые переменные

$$
\eta_{2 j-1}=\frac{\xi_{2 j-1}+\imath \xi_{2 j}}{\sqrt{2}}, \quad \eta_{2 j}=\frac{\xi_{2 j-1}-\imath \xi_{2 j}}{\sqrt{2}}, \quad j=1, \ldots,\left[\frac{n}{2}\right] .
$$

Тогда $\eta_{2 j-1} \eta_{2 j}$ - градуируюшие элементы, и их использование уменьшает количество рассматриваемых случаев. Мы не стали этого делать по следующим причинам:

1) если $n$ нечетно, то введение градуирующих элементов все равно не позволяет рассматривать только случай нулевого веса;

2) уменьшение количества случаев компенсируется большей сложностью их рассмотрения (формула композиции в переменных $\eta$ сложнее, чем в переменных $\xi$ ). 
ДоКАЗАТЕЛЬСТво ТЕОРЕМЫ 3. МЫ долЖны доказать, что

$$
\left.h\left(h\left(E_{1}, E_{2}\right), E_{3}\right) \pm h\left(h\left(E_{2}, E_{3}\right), E_{1}\right) \pm h\left(h\left(E_{3}, E_{1}\right), E_{2}\right)\right)=0,
$$

где знаки определяются четностями $E_{1}, E_{2}$ и $E_{3}$. Пусть $E_{1}=F_{1} F_{2} F_{3} E^{\prime}, E_{2}=$ $F_{1} F_{2} F_{4} E^{\prime \prime}, E_{3}=F_{1} F_{3} F_{4} E^{\prime \prime \prime}$, где $\left|F_{1}\right|+\left|F_{2}\right|$ нечетно и $\left|F_{3}\right|+\left|F_{4}\right|$ нечетно. Пусть $\left|E^{\prime}\right|=a$, $\left|E^{\prime \prime}\right|=b,\left|E^{\prime \prime \prime}\right|=c,\left|F_{1}\right|=p,\left|F_{2}\right|=q,\left|F_{3}\right|=r,\left|F_{4}\right|=s$. Пусть $p$ нечетно. Надо рассмотреть два случая:

a) $r$ нечетно;

б) $r$ четно.

Мы рассмотрим случай а), так как случай б) рассматривается аналогично:

$$
\begin{aligned}
h\left(h\left(E_{1}, E_{2}\right), E_{3}\right) & =t^{(p+q-1) / 2} h\left(c_{(p+q-1) / 2}\left(E_{1}, E_{2}\right), E_{3}\right) \\
& =(-1)^{a} t^{(p+q-1) / 2} h\left(F_{3} E^{\prime} F_{4} E^{\prime \prime}, F_{1} F_{3} F_{4} E^{\prime \prime \prime}\right) \\
& =(-1)^{a+1} t^{(p+q+r+s-2) / 2} c_{(r+s-1) / 2}\left(F_{3} F_{4} E^{\prime} E^{\prime \prime}, F_{3} F_{4} F_{1} E^{\prime \prime \prime}\right) \\
& =(-1)^{b} t^{(p+q+r+s-2) / 2} E^{\prime} E^{\prime \prime} F_{1} E^{\prime \prime \prime} \\
& =(-1)^{a} t^{(p+q+r+s-2) / 2} F_{1} E^{\prime} E^{\prime \prime} E^{\prime \prime \prime} .
\end{aligned}
$$

В этом случае $h\left(E_{3}, E_{1}\right)=0$, поэтому надо вычислить $h\left(h\left(E_{2}, E_{3}\right), E_{1}\right)$. Перенос $E_{1}$ на последнее место приводит к умножению на $(-1)^{a(b+c+1)}$. Имеем

$$
\begin{aligned}
& (-1)^{a(b+c+1)} h\left(h\left(F_{1} F_{2} F_{4} E^{\prime \prime}, F_{1} F_{3} F_{4} E^{\prime \prime \prime}\right), F_{1} F_{2} F_{3} E^{\prime}\right) \\
& \quad=(-1)^{a(b+c+1)} t^{(p+s-1) / 2} h\left(c_{(p+s-1) / 2}\left(F_{1} F_{4} F_{2} E^{\prime \prime}, F_{1} F_{4} F_{3} E^{\prime \prime \prime}\right), F_{1} F_{2} F_{3} E^{\prime}\right) \\
& \quad=(-1)^{a(b+c+1)+b+1} t^{(p+s-1) / 2} h\left(F_{2} E^{\prime \prime} F_{3} E^{\prime \prime \prime}, F_{1} F_{2} F_{3} E^{\prime}\right) \\
& \quad=(-1)^{a(b+c+1)} t^{(p+q+r+s-2) / 2} c_{(q+r-1) / 2)}\left(F_{2} F_{3} E^{\prime \prime} E^{\prime \prime \prime}, F_{2} F_{3} F_{1} E^{\prime}\right) \\
& \quad=(-1)^{(a(b+c+1)+b+c+1} t^{(p+q+r+s-2) / 2} E^{\prime \prime} E^{\prime \prime \prime} F_{1} E^{\prime} \\
& =(-1)^{a+1} t^{(p+q+r+s-2) / 2} F_{1} E^{\prime} E^{\prime \prime} E^{\prime \prime \prime}
\end{aligned}
$$

т.е. сумма равна 0 .

\section{СПИСОК ЦИТИРОВАННОЙ ЛИТЕРАТУРЫ}

[1] Van der Hijligenberg N., Post G. Defining relations for Lie algebras of vector fields // Indag. Math. (N.S.). 1991. № 2. P. 207-218.

[2] Кочетков Ю. Ю. Деформации гамильтоновой алгебры $H(2) / /$ Функцион. анализ и его прилож. 1993. Т. 28. № 3. С. 77-79.

[3] Van der Hijligenberg N., Kotchetkov Yu., Post G. Deformations of $S(0, n)$ and $H(0, n)$ // Internat. J. Algebra Comput. 1993. V. 1. P. 57-77.

[4] Fialowski A. On the deformations of $L_{1} / /$ Studia Sci. Math. Hungar. 1985. V. 20. P. 433-438.

[5] Кочетков Ю. Ю., Пост Г. Ф. Деформации бесконечномерной нильпотентной алгебры Ли $L_{2} / /$ Функцион. анализ и его прилож. 1992. Т. 26. №4. С. 90-92.

[6] Van der Hijligenberg N., Kotchetkov Yu. The absolute rigidity of the Neveu-Schwarz and Ramond superalgebras // J. Math. Phys. 1996. V. 37. №11. P. 5858-5868.

Институт новых технологий, г. Москва 